2

3

4

5

6

7

8

9 \section{.} (8)

\title{
Application of the half decimal place rule to increase the peptide identification rate
}

Christian J Koehler, Ravi Chand Bollineni and Bernd Thiede*

Department of Biosciences, University of Oslo, Oslo, Norway

Keywords: data-dependent acquisition, half decimal place rule, mass defect, mass spectrometry, peptide spectrum matches

*To whom the correspondence should be addressed:

Bernd Thiede, Department of Biosciences, University of Oslo, P.O. Box 1066 Blindern, 0316 Oslo, Norway, Tel.: +47-22840533; Fax +47-22840501; E-mail: bernd.thiede@ibv.uio.no

Abbreviations: DDA, data-dependent acquisition; HDPR, half decimal place rule; LC, liquid chromatography; MS, mass spectrometry; PSM, peptide spectrum match; UHPLC, ultra high performance liquid chromatography 


\section{Abstract}

2 RATIONALE: Many MS2 spectra in bottom-up proteomics experiments remain unassigned.

3 To improve proteome coverage, we applied the half decimal place rule (HDPR) to remove 4 non-peptidic molecules. The HDPR considers the ratio of the digits after the decimal point to 5 the full molecular mass and results in a relatively small permitted mass window for most 6 peptides.

7

8 METHODS: First, the HDPR mass filter was calculated for the human and other proteomes.

9 Subsequently, the HDPR was applied to three technical replicates of an in-solution tryptic digest of HeLa cells which were analysed by LC-MS using a quadrupole-orbitrap mass spectrometer ( $Q$ Exactive). In addition, the same sample was analysed three times with a fixed exclusion list. The exclusion list was based on only choosing doubly charged ions for fragmentation.

RESULTS: The peptide spectrum match (PSM) rate increased by $2-4 \%$ applying HDPR filters from 0.1-0.25 Da and 75-150 ppm, respectively. Excluding all MS2 events by applying an HDPR filter of doubly charged ions, we were able to improve PSMs by $0.9 \%$ and the PSM rate by $2.5 \%$.

CONCLUSIONS: An algorithm to filter precursors based on the HDPR was established to improve the targeting of the acquisition of MS2 spectra in data-dependent acquisition (DDA) experiments. According to our data, a total gain of PSMs of $1-5 \%$ might be achievable if the HPDR filter would already be applied during MS data acquisition. 


\section{Introduction}

Data-dependent acquisition (DDA) is an operation mode in mass spectrometry predominantly used in discovery proteomics where algorithms decide which precursor is subjected to further fragmentation and MS2 acquisition. The target is typically not known and the aim is to acquire spectra from as many different peptides as possible to obtain a map of the sample that is as complete as possible. Typically, the DDA algorithm ranks peaks in an MS spectrum by intensity. Dynamic exclusion of already taken precursors is used to maximise the peptide identification rate. Furthermore, additional rules can be specified to include or exclude possible precursors based on the charge state, minimum intensity and mass range of the precursor. Nevertheless, undersampling of complex proteomic samples remains one of the major issues in DDA-based proteomics. Furthermore, a significant proportion of MS2 spectra in bottom-up proteomics experiments remain unassigned despite significant improvements of acquisition speed and resolution of mass spectrometers during the last years. These unexplained MS2 spectra might originate from e.g., unexpected posttranslational modifications, chemical modifications, sequencing errors, unknown alternative splice variants and polymorphisms ${ }^{[1]}$. Another reason might be that the analysed molecules are not peptides and thus worthless for proteome analyses. Actually, it was observed that up to $30 \%$ of detected molecules can be of nonpeptidic origin ${ }^{[2]}$.

The mass defect is the difference in mass between an atom and the sum of the masses of the protons and neutrons. Each isotope has a unique mass defect with carbon-12 (zero mass defect), hydrogen-1 (0.0078), nitrogen-14 (0.0031), oxygen-16 (-0.0051) and sulfur-32 ($0.0279)^{[3]}$. The mass distribution of the theoretically possibly tryptic peptides revealed gaps and low-populated areas [4] [5] [6] [7] [8]. Furthermore, the statistical occurrences of average amino acids with $\mathrm{C}_{4.9384} \mathrm{H}_{7.7583} \mathrm{~N}_{1.3577} \mathrm{O}_{1.4773} \mathrm{~S}_{0.0417}{ }^{[9]}$, results in a slightly increased mass defect with increased molecular mass. It has been demonstrated that the mass defect can be used to improve identification of proteins by peptide mass fingerprinting using MALDI-TOF [10] [11] and MALDI-FTICR ${ }^{[12]}{ }^{[13]}$ excluding non-peptide signals. A model of peptide mass cluster centres was developed, considering amino acid frequencies, average length of proteins in the database, and cleavage specificity of endoproteinases, and the distance to the nearest cluster was used to calibrate peptide peak lists and to identify non-peptide peaks [14]. Furthermore, it can be used to distinguish modified from unmodified peptides as shown for phosphopeptides ${ }^{[15]}$, glycopeptides ${ }^{[16]}$ and oligonucleotide-peptide cross-links ${ }^{[17]}$. A nonlinear Random Forest classification and a discrete mapping approach were developed to increase fragmentation efficiency on-the fly for specific subsets of analytes such as shown for phosphorylated and iodinated peptides ${ }^{[18]}$. Moreover, the HDPR was defined to take advantage of the mass defect ${ }^{[10]}$. It is based on the observation that the first digit of the 
mantissa of the mass of a peptide is near the half of the first digit of mass values between 500 and 999 and near the half of the first two digits of mass values between 1000 and 1999 , and near the half of the first digit of the peptide mass in the mass range 2000 to $3000{ }^{\text {[10] [11]. }}$ This means that for peptides with molecular masses between 100 and $999 \mathrm{Da}$, the mantissa is typically between 0.05 (at $100 \mathrm{Da}$ ) and 0.5 (at $999 \mathrm{Da}$ ), and for peptides with molecular masses between 1000 and $1999 \mathrm{Da}$, the mantissa is typically between 0.5 and 0.99 . The main application of the HDPR is so far on peptide mass fingerprints acquired by MALDI-MS to filter chemical contaminants and to quickly evaluate instrument calibration.

In this report, we performed a new calculation of the HDPR. The HDPR was applied to a complex proteome derived from HeLa cells analysed by LC-MS to select precursors subjected to MS2 acquisition with the aim to increase the peptide spectrum identification rate.

\section{Materials and Methods}

\section{Cell culture}

HeLa cells were grown as a monolayer in RPMI-1640 supplemented with $10 \%$ fetal bovine serum and maintained in a humid incubator at $37^{\circ} \mathrm{C}$ in a $5 \% \mathrm{CO}_{2}$ environment to reach a density of $1 \times 10^{5}$ cells $/ \mathrm{ml}$. Cell pellets were frozen and stored in liquid nitrogen. Pellets of cells were thawed on ice and $800 \mu \mathrm{l}$ SILAC Phosphoprotein lysis buffer B (Invitrogen, Oslo, Norway) was added. The cell slurry was homogenized with a pestle (20x) for mechanical breakage of the cells followed by sonication using an Ultrasonic processor (UP400s, Dr. Hielscher). Samples were centrifuged at $16,000 \mathrm{~g}$ for 20 minutes at $4^{\circ} \mathrm{C}$ in a Heraeus Biofuge pico (Kendro, Hanau, Germany) and the supernatant was aliquoted in $40 \mu$ l aliquots.

\section{In-solution digestion}

To one HeLa cell aliquot, $400 \mu \mathrm{l}$ of ice cold acetone/methanol (1:1) acidified with $0.1 \%$ hydrochloric acid $(\mathrm{v} / \mathrm{v})$ was added, vortexed and precipitated at $-20^{\circ} \mathrm{C}$ over night. Samples were centrifuged at $16,000 \mathrm{~g}$ for 20 minutes at $4^{\circ} \mathrm{C}$ (Heraeus Biofuge pico) and the supernatant was discarded. Proteins were re-dissolved in $50 \mu \mathrm{l} 6 \mathrm{M}$ urea and $100 \mathrm{mM}$ ammonium bicarbonate, $\mathrm{pH}$ 7.8. For reduction and alkylation of cysteines, $5 \mu \mathrm{l}$ of $200 \mathrm{mM}$ DTT was added and the samples were incubated at $37^{\circ} \mathrm{C}$ for $1 \mathrm{~h}$ followed by addition of $20 \mu \mathrm{l}$ $200 \mathrm{mM}$ iodoacetamide for $1 \mathrm{~h}$ at room temperature in the dark. The alkylation reaction was quenched by adding $20 \mu \mathrm{l} 200 \mathrm{mM}$ DTT. The proteins were digested with trypsin in a final volume of $225 \mu \mathrm{l}$ for $16 \mathrm{~h}$ at $37^{\circ} \mathrm{C}$. The digestion was stopped by adding $100 \mu \mathrm{l} 1 \%$ formic acid and the generated peptides were purified using a Strata C18-E SPE column 
1 (Phenomenex, Værløse, Denmark), and dried using a Speed Vac concentrator (Eppendorf,

2 Hamburg, Germany).

\section{Liquid chromatography-mass spectrometry (LC-MS)}

Three technical replicates tryptic digest of the HeLa proteome were analyzed using an Ultimate 3000 nano-UHPLC system (Dionex, Sunnyvale, CA, USA) connected to a $Q$ Exactive mass spectrometer (ThermoElectron, Bremen, Germany) equipped with a nano electrospray ion source. For liquid chromatography separation, an Acclaim PepMap 100 column (C18, $3 \mu \mathrm{m}$ beads, $100 \AA, 75 \mu \mathrm{m}$ inner diameter) (Dionex, Sunnyvale CA, USA) capillary of $50 \mathrm{~cm}$ bed length was used. A flow rate of $300 \mathrm{~nL} / \mathrm{min}$ was employed with a solvent gradient $3-35 \% \mathrm{~B}$ in $97 \mathrm{~min}$, to $50 \% \mathrm{~B}$ in $13 \mathrm{~min}$ and then to $80 \% \mathrm{~B}$ in $2 \mathrm{~min}$. Solvent A was $0.1 \%$ formic acid and solvent $B$ was $0.1 \%$ formic acid $/ 90 \%$ acetonitrile.

The mass spectrometer was operated in the data-dependent mode to automatically switch between MS and MS2 acquisition. Survey full scan MS spectra (from m/z 400 to 2000) were acquired with the resolution $R=70,000$ at $\mathrm{m} / \mathrm{z} 200$, after accumulation to a target of $1 \mathrm{e} 6$. The maximum allowed ion accumulation times were $100 \mathrm{~ms}$. The method used allowed sequential isolation of up to the ten most intense, doubly charged ions, depending on signal intensity (intensity threshold 1.7e4), for fragmentation using higher-energy collision induced dissociation (HCD) at a target value of 10,000 charges and a resolution $R=17,500$. Only double charged precursors were considered for MS2 to assure comparability between different experiments. The peptide match filter was set to off. Target ions already selected for MS/MS were dynamically excluded for $30 \mathrm{sec}$. The isolation window was $\mathrm{m} / \mathrm{z}=2$ without offset. The maximum allowed ion accumulation for the MS/MS spectrum was $60 \mathrm{~ms}$. For accurate mass measurements, the lock mass option was enabled in MS mode and the polydimethylcyclosiloxane ions generated in the electrospray process from ambient air were used for internal recalibration during the analysis.

\section{Data analysis}

Data were acquired using Xcalibur v2.5.5 and raw files were processed to generate peak list in Mascot generic format ( ${ }^{*}$.mgf) using ProteoWizard release version 3.0.7230. Database searches were performed using Mascot in-house version 2.4.0 to search the SwissProt database (Human, 21.01.2016, 20187 proteins) assuming the digestion enzyme trypsin, at maximum one missed cleavage site, fragment ion mass tolerance of $0.05 \mathrm{Da}$, parent ion tolerance of $10 \mathrm{ppm}$ and oxidation of methionines, and acetylation of the protein $\mathrm{N}$-terminus as variable modifications. Scaffold (version Scaffold_4.4.3, Proteome Software Inc., Portland, OR) was used to validate MS2 based peptide and protein identifications. Peptide 
1 identifications were accepted if they could be established at greater than $95.0 \%$ probability

2 by the Scaffold Local FDR algorithm. Protein identifications were accepted if they could be

3 established at greater than $99.9 \%$ probability. The mass spectrometry proteomics data have

4 been deposited to the ProteomeXchange Consortium ${ }^{[19]}$ via the PRIDE partner repository

5 with the dataset identifier PXD004900 (Username: reviewer91076@ebi.ac.uk; password:

6 OEdGEubA).

7

\section{Results and Discussion}




\section{Calculation of the denominator for the HDPR mass filter of the human proteome}

An in silico tryptic digest without missed cleavages of the reviewed human Swiss-Prot proteome database was performed. From the resulting set of all human tryptic peptides, a subset was created using only peptides with a minimum number of five amino acids per peptide and all peptides smaller than $600 \mathrm{Da}$ or larger than 4,000 Da were disregarded. Assuming a minimum charge of $2+$, this subset contains 639,699 of useful detectable peptides in a mass spectrometry experiment utilizing a $300-2,000 \mathrm{~m} / \mathrm{z}$ scan range. All further calculations were based on this set of human tryptic peptides. Notably, certain posttranslational modifications (PTMs), such as glycosylation and phosphorylation lead to a significant change of the HDPR ratio in comparison to corresponding non-modified peptides. For PTM-specific experiments, the HDPR filter might be changed accordingly to improve the selection of modified peptides for fragmentation.

Every individual peptide has its own ideal denominator where the distance between the calculated mantissa and the real mantissa becomes zero. For a proteomics experiment, the best denominator for the HDPR rule is where the average of all peptides share a minimal distance. Therefore, we calculated the denominator for which the difference between the calculated and actual mantissa is minimized for each peptide of the human tryptic peptidome. Because every peptide mass has several minima (Fig. 1A and $1 \mathrm{~B}$ ), the value nearest to the original, previous observed denominator of 2000 was used as the optimal denominator. The average of all these denominators of the whole human tryptic peptidome was 2007 without and 2011 with carbamidomethylation (Fig. 2A). Other organisms revealed similar values using the SwissProt database, e.g., 2010 for mouse (533,985 tryptic peptides), 2005 for Arabidopsis thaliana (374,786 tryptic peptides), 2008 for rat (227,089 tryptic peptides), 1991 Schizosaccharomyces pombe (139,510 tryptic peptides), 2005 for bovine (139,188 tryptic peptides), 1990 for Dictyostelium discoideum (122,496 tryptic peptides), 2009 for Drosophila melanogaster (110,397 tryptic peptides), and 2019 for Caenorhabditis elegans $(107,559$ tryptic peptides).

\section{Application of the HDPR filter after data acquisition}

To assess the HDPR algorithm, three technical replicates of a complex proteome derived from an in-solution digest of HeLa cells were analysed using two hours LC runs connected to a quadrupole orbitrap mass spectrometer ( $Q$ Exactive). After conventional processing, the resulting peak list (mgf format) was further processed with an in-house python script filtering all MS2 spectra based on the HDPR rule, resulting in peak lists which contained only peptides in which the distance of the true and the theoretical mantissa were below the defined threshold. In this way, we simulated if a MS2 spectrum would have been acquired 
1 (passed the HDPR) or rejected. The unfiltered and HDPR filtered mgf files were searched using Mascot. This approach consequently results in a reduction of searchable MS2 spectra and therefore the loss of peptide spectrum matches (PSMs). However, the relation of the filtered out not-matching and matching spectra will give a measure of the quality of the HDPR algorithm. We compared different filter thresholds, both fixed mass filters allowing a fixed distance between the mantissae $(0.1 \mathrm{Da}, 0.15 \mathrm{Da}, 0.2 \mathrm{Da}$ and $0.25 \mathrm{Da}$ ) as well as proportional mass filters with a distance based on the acquired peptide mass (75 ppm, 100 ppm, 125 ppm, 150 ppm) (Table 1).

With more restrictive filters, the identified number of proteins and number of PSMs decreased (3,111 to 3,006 for $\mathrm{Da}$ and 3,109 to 3,040 for ppm, respectively), as less MS2 spectra were available. However, the identification rate (PSMs/MS2 spectra (\%)) increased from $51.3 \%$ to $52.7 \%$ for $\mathrm{Da}$ and $50.8 \%$ to $52.5 \%$ for ppm, respectively (Table $1 \mathrm{~B}$ ). Notably, it was always higher than in the original dataset with $48.7 \%$ (Table $1 \mathrm{~A}$ ).

Considering only the rejected data (Total - HDPR), a distinct lower identification rate (PSMs/MS2 spectra (\%); $4.2 \%-34.3 \%$ for Da and $5.6 \%-28.7 \%$ for ppm) than for the unfiltered data $(48.7 \%)$ (Table $1 \mathrm{~A})$ was obtained. This result shows that more non-matching MS2 spectra were removed in comparison to the unfiltered data set.

Based on the identification rate for each filter, a maximal gain of PSMs can be calculated (Table 1D). This gain is a theoretical calculation and based on the assumption that acquired precursors which did not pass the HDPR would be rejected and replaced with the next precursor in the DDA algorithm which passes the HDPR. To calculate the maximal gain of PSMs, precursors which fail the HDPR but result in a PSM need to be subtracted.

As an example, using an HDPR mass filter of $0.15 \mathrm{Da}, 33,519$ precursors remained which led to 17,535 PSMs (52.3\% identification rate) (Table 1B). As a consequence, 3,795 MS2 spectra were discarded, of which 641 MS2 spectra led to PSMs (Table 1C). Hence, the filter incurred the loss of $83.1 \%$ of non-usable precursors and of $16.9 \%$ of peptide precursors (1.7\% of all MS2 spectra) (Table $1 \mathrm{C}$ ). In case of an MS instrument operating with an HDPR filter, all discarded MS2 spectra would give time to acquire MS2 spectra with an increased chance of peptide identification. If the 3,795 filtered precursors with HDPR of 0.15 Da would have been used to acquire MS2 spectra with an identification rate of $52.3 \%$, theoretically 1,985 MS2 spectra would be identified. Subtracting the 641 peptide identifications which were discarded by the algorithm, a theoretical total gain of 1,345 additional peptides can be obtained with HDPR filter $0.15 \mathrm{Da}$, which corresponds to a gain of $7.4 \%$ of total PSMs (Table 1D). However, the theoretical gain is a too high estimation as less intense precursors will be chosen for MS2 fragmentation (top10 + n peaks) and not all MS2 spectra which led initially to an identification event will be replaced by a PSM. The more stringent the HDPR filter is 
applied, the more precursors are disregarded and the chance to supplement these free with a peptide matching HDPR precursor becomes increasingly difficult. A more relaxed filter on the other hand leads to a smaller gain. The optimal values have to be determined experimentally and depend highly on the sample complexity and duty cycle.

Apart from the experimental determination of the optimal HDPR filter stringency, a theoretical approach of a useful threshold was considered. Using all theoretical peptides from the human database, we calculated the threshold where the distance of theoretical and true mantissae includes $95 \%$ of the human tryptic peptides using the determined optimal HDPR ratio of 2011 (Fig. 2B). As a result, a distance of $+/-0.1497 \mathrm{Da}$ for the fixed mass filter and $102 \mathrm{ppm}$ for the proportional mass filter, respectively, were calculated. A total deviation of +/$0.15 \mathrm{Da}$ and +/- $100 \mathrm{ppm}$, respectively, was found to be a suitable approximation (Fig. $3 \mathrm{~A}$ and $3 B$ ).

The use of a proportional mass filter would be dependent on the mass of each peptide in contrast to the fixed mass filter. Using 100 ppm, on average a theoretical total gain of 1,241 peptide matches $(6.8 \%$ of total peptide identifications) was calculated, consistent for all three replicates (Table 1D). Using $100 \mathrm{ppm}$, the filtering effects were slightly more moderate than using the fixed mass filter of $0.15 \mathrm{Da}$. While the fixed mass filter is biased towards small masses being more stringent to high masses, the reverse is true for the proportional mass filter (Fig. 3C, 3D, 3E). Covering approximately 95\% of the tryptic proteome, a fixed mass filter of $0.15 \mathrm{Da}$ resulted in a higher gain in PSMs than a $100 \mathrm{ppm}$ proportional mass filter. A probable explanation is the discrepancy between the theoretical calculation and the experimental higher abundance of low mass peptides of the peptides being available for MS and the just noted preference.

Notably, the ideal tolerance is dependent on the peptide mass, with a higher spread for higher masses ${ }^{[10]}$ (Fig. 3B). Based on the HDPR ratio 2011, the tolerable deviation can be narrowed to $+/-0.1323 \mathrm{Da}$ to cover $95 \%$ of the human tryptic peptidome if only masses less than 3,000 Da are considered. For peptides less than 2,000 Da, even a deviation of 0.1130 Da would be enough whereas a deviation of $+/-0.2736 \mathrm{Da}$ is required to include $95 \%$ of the peptides between 3,000 $\mathrm{Da}$ and 4,000 $\mathrm{Da}$.

\section{Application of the HDPR filter during data acquisition}

We were not able to change the acquisition software of our mass spectrometer to implement an HDPR filter. However, we applied an exclusion list to demonstrate the feasibility of this filter. Due to the linear dependency of the mantissa from the mass defined by the HDPR rule and a fixed tolerance of e.g., +/- $0.15 \mathrm{Da}$ (Figure $3 \mathrm{~A}$ ), it is possible to create an exclusion list which contains the masses between consecutive HDPR masses. These masses have a fixed 
tolerance which fills the gap between accepted mass areas (Figure 4). Thus, exclusion lists can be defined with an absolute tolerance. As the tolerance depends on the charge state, it is only possible to create an exclusion list for one charge state at a time and for the linear mass filter only. For this reason, all experiments had been carried out allowing only doubly charged precursors to be considered for MS2 fragmentation. First, we implemented an exclusion list based on the HDPR tolerance +/- $0.15 \mathrm{Da}$, acquired three technical replicates and compared them against three replicates acquired without exclusion list and were able to improve the PSM rate, but the absolute number of PSMs was not improved (Table 1E). Therefore, we used a more relaxed exclusion list based on the HDPR tolerance of +/- 0.20 Da and repeated the experiment. Without exclusion, on average 37,315 MS2 spectra were acquired resulting in 18,034, 18,146 and 18,346 PSMs per replicate (on average 18,175 \pm 129) (Table $1 \mathrm{~A}$ ). Using the exclusion list, on average 35,782 MS2 spectra were acquired leading to $18,329,18,373$, and 18,413 PSMs per replicate (on average $18,342 \pm 74)$. The identification rate increased by $2.6 \%$ ( $51.3 \%$ vs. $48.7 \%)$ because 1,533 less MS2 spectra were acquired but 167 more PSMs were identified (Table 1E). The experimental gain appears to be moderate compared to the theoretical gain with only $0.9 \%$ increase in total PSMs. A similar gain in protein identification has been achieved with peptide mass fingerprinting using MALDI-TOF instrumentation ${ }^{[14]}$. Still, a comparison is difficult because removing non-peptide peaks from peptide mass fingerprints is performed postacquisition and applied to single proteins. Anyhow, we were able to increase the total PSMs simply by the application of the HDPR implemented as a fixed exclusion list. Nevertheless, we could only compare acquisitions based on one charge state which limits the number of available precursors. In the experiment using the exclusion list, the frequency of recording of ten consecutive MS2 spectra was decreased compared to normal acquisitions. Furthermore, we could only compare acquisitions with the filter turned on (exclusion list) or off (normal acquisition). If it had been possible to apply the filter as long as there are precursors to choose from and otherwise continue with less favourable precursors, the PSMs would have been increased more without negative consequences. Ultimately, the benefit of the HDPR filter in a real-time decision tree is difficult to predict without an actual implementation.

If the HDPR filter was be implemented into the acquisition software, it must observe $\mathrm{m} / \mathrm{z}$ values, determine the charge, and calculate the actual mass, all is typically included into current mass spectrometry software. Apart from that, a lookup table of allowed and disallowed mass regions could be calculated based on the parameters "denominator" and the tolerance in $\mathrm{Da}$ or ppm. 


\section{Conclusions}

2 We developed a simple algorithm to filter precursors based on the idea of HDPR in order to

3 improve the targeting of the acquisition of MS2 spectra in DDA experiments and to increase

4 the number of peptide identifications amongst the chosen precursors. We were not able to

5 implement the filter into the acquisition parameters of our mass spectrometer, but have

6 shown the feasibility by an exclusion list applied to the same samples. According to our data,

7 a total gain of PSMs of $1-5 \%$ might be achievable by including HDPR-based filtering in MS

8 acquisition for standard peptide identification. This gain would just require changes in the MS

9 acquisition software. However, the HDPR-based filter should be optional because it might not 10 be suitable for the analysis of certain modified peptides which lead to relative large variations 11 of the HDPR denominator such as phosphorylated, glycosylated, and lipidated peptides. 12 Finally, the HDPR ratio must be determined empirically, because PSMs of precursors which do not fulfil the HDPR filter must be replaced with low intensity MS2 spectra, which cannot be theoretically predicted.

Acknowledgements: Financial support from the Norwegian Cancer Society (Project 4514636) is gratefully acknowledged. 


\section{Figure legends}

\section{Figure 1: Graphical representation of the determination of HDPR ratios.}

Graphical representations of the iterative calculations of two peptides with different masses ( $\mathrm{m} / \mathrm{z} 815.4137$ and $\mathrm{m} / \mathrm{z} 2442.2763$ ) using formula 1.2 are displayed in $A$ and $B$, respectively. The denominator is displayed on the $x$-axis, and the mantissa (top) or distance (bottom) is shown on the y-axis. The real decimal place of the peptide masses is graphed as a horizontal dashed line. The higher the mass the more often the calculated decimal place traverses the real decimal place (top, A vs. B). The distance between the calculated and real decimal place becomes zero whenever the function traverses the true decimal place ( $A$ and $\mathrm{B}$, bottom). To calculate the HDPR ratio, the denominator creating a minimal distance to the real decimal place and being closest to 2000 was calculated. For the two presented examples, these values are indicated by arrows and resulted in HDPR ratios of 1971 (A) and 1913 (B).

Figure 2: Histogram of the frequency of HDPR ratios and distances of the calculated mantissae.

The HDPR ratio was determined for all human tryptic peptides and the distribution of the HDPR ratios is shown in A. The resulting average HDPR ratio was $2011.95 \%$ of all tryptic peptides with a minimum of five amino acids, and 600 to $4000 \mathrm{Da}$ are within a tolerance of $0.1497 \mathrm{Da}$ (light grey) using this HDPR ratio (B).

Figure 3: The mantissae and frequency of the human tryptic peptides in dependence of molecular mass.

The mantissae vs. masses are shown. The greyscale of the dots indicates the frequency. The HDPR filter of $0.15 \mathrm{Da}(\mathrm{A})$ and $100 \mathrm{ppm}(\mathrm{B})$, respectively, are shown by black lines. The frequency of all peptides $(C)$, peptides deviating more than $0.15 \mathrm{Da}(\mathrm{D})$, and peptides deviating more than 100 ppm (E) are depicted using HDPR ratio 2011. A bias towards small masses can be observed for the proportional mass filter (ppm) and towards high masses for the fixed filter (Da).

\section{Figure 4: Implementation of the linear HDPR filter by using an exclusion list.} HDPR masses can be calculated based on equation 1.2. In a linear HDPR filter, HDPR masses with their tolerances create accepted mass areas which match the HDPR (bright area). Consequently, the areas in between are non-accepted mass areas with a central mass defined in the exclusion list to implement the HDPR filter (dark gray). Here, uncharged 
1 masses are displayed. For a filter, the uncharged masses and the tolerances have to be

2 converted in $\mathrm{m} / \mathrm{z}$ values for every charge. Therefore, the tolerances vary with charge and

3 only one charge state can be defined in an exclusion list to create a HDPR filter.

4

5

6 


\begin{tabular}{|c|c|c|c|c|c|}
\hline & \begin{tabular}{|l|} 
HDPR \\
filter
\end{tabular} & \begin{tabular}{|l|} 
Protein \\
ID
\end{tabular} & \begin{tabular}{|l|} 
MS2 \\
spectra (\%)
\end{tabular} & PSMs (\%) & $\begin{array}{l}\text { PSM/MS2 } \\
\text { spectra [\%] }\end{array}$ \\
\hline A. Total & no & 3180 & 37315 & 18175 & 48.7 \\
\hline \multirow[t]{8}{*}{ B. HDPR } & $0.10 \mathrm{Da}$ & 3006 & 29189 & 15391 & 52.7 \\
\hline & $0.15 \mathrm{Da}$ & 3088 & 33519 & 17535 & 52.3 \\
\hline & $0.20 \mathrm{Da}$ & 3108 & 34768 & 17999 & 51.8 \\
\hline & $0.25 \mathrm{Da}$ & 3111 & 35230 & 18089 & 51.3 \\
\hline & 75 ppm & 3040 & 31339 & 16458 & 52.5 \\
\hline & $100 \mathrm{ppm}$ & 3088 & 33763 & 17568 & 52.0 \\
\hline & $125 \mathrm{ppm}$ & 3099 & 34972 & 17946 & 51.3 \\
\hline & $150 \mathrm{ppm}$ & 3109 & 35579 & 18078 & 50.8 \\
\hline \multirow[t]{8}{*}{ C. Total - HDPR (\%) } & $0.10 \mathrm{Da}$ & -174 & $8126(21.8)$ & $2784(15.3)$ & 34.3 \\
\hline & $0.15 \mathrm{Da}$ & -92 & $3796(10.2)$ & $640(3.5)$ & 16.9 \\
\hline & $0.20 \mathrm{Da}$ & -72 & $2547(6.8)$ & $176(1.0)$ & 6.9 \\
\hline & $0.25 \mathrm{Da}$ & -69 & $2085(5.6)$ & $86(0.5)$ & 4.2 \\
\hline & $75 \mathrm{ppm}$ & -140 & $5976(16.0)$ & $1717(9.4)$ & 28.7 \\
\hline & $100 \mathrm{ppm}$ & -92 & $3552(9.5)$ & 607 (3.3) & 17.1 \\
\hline & $125 \mathrm{ppm}$ & -81 & $2343(6.3)$ & $229(1.3)$ & 9.8 \\
\hline & $150 \mathrm{ppm}$ & -71 & $1736(4.7)$ & $97(0.5)$ & 5.6 \\
\hline \multirow[t]{8}{*}{ D. Max.gain } & $0.10 \mathrm{Da}$ & & & $1501(8.3)$ & \\
\hline & $0.15 \mathrm{Da}$ & & & $1345(7.4)$ & \\
\hline & $0.20 \mathrm{Da}$ & & & $1142(6.3)$ & \\
\hline & $0.25 \mathrm{Da}$ & & & $984(5.4)$ & \\
\hline & $75 \mathrm{ppm}$ & & & $1421(7.8)$ & \\
\hline & $100 \mathrm{ppm}$ & & & $1241(6.8)$ & \\
\hline & $125 \mathrm{ppm}$ & & & $973(5.4)$ & \\
\hline & $150 \mathrm{ppm}$ & & & $785(4.3)$ & \\
\hline \multirow[t]{2}{*}{ E. Total with exclusion } & $0.15 \mathrm{Da}$ & 3172 & 35224 & 18022 & 51.2 \\
\hline & $0.20 \mathrm{Da}$ & 3176 & 35782 & 18342 & 51.3 \\
\hline
\end{tabular}

\section{Table 1: The effect of the HDPR filter}

Average numbers of three technical replicates are shown. The results obtained without HDPR filter (A), and with different HDPR filters (B) are presented. In $C$, the difference between $A$ and $B$ is displayed. Furthermore, the maximal theoretical gain in PSMs is shown in $\mathrm{D}$. The data obtained after using an exclusion list for all doubly charged ions is shown in $\mathrm{E}$. Protein ID, number of identified proteins; ID, number of identifications; Max. gain = MS2 spectra (Total - HDPR) x PSMs/MS2 spectra (HDPR) - PSMs (Total - HDPR). 


\section{References}

[1] J. M. Chick, D. Kolippakkam, D. P. Nusinow, B. Zhai, R. Rad, E. L. Huttlin, S. P. Gygi, Nat Biotechnol 2015, 33, 743.

[2] A. Michalski, J. Cox, M. Mann, J Proteome Res 2011, 10, 1785.

[3] L. Sleno, J Mass Spectrom 2012, 47, 226.

[4] M. Mann, in Annual Conference on Mass Spectrometry and Allied Topic, American Society of Mass Spectrometry: Atlanta, GA, 1995.

R. A. Zubarev, P. Håkansson, B. Sundqvist, Anal Chem 1996, 68, 4060.

[7] A. V. Nefedov, I. Mitra, A. R. Brasier, R. G. Sadygov, J Proteome Res 2011, 10, 4150.

[8] I. Mitra, A. V. Nefedov, A. R. Brasier, R. G. Sadygov, Anal Chem 2012, 84, 3026.

[9] M. W. Senko, S. C. Beu, F. W. McLafferty, J Am Soc Mass Spectrom 1995, 6, 229.

[10] F. Schmidt, M. Schmid, P. R. Jungblut, J. Mattow, A. Facius, K. P. Pleissner, J Am Soc Mass Spectrom 2003, 14, 943.

[11] J. A. Karty, M. M. Ireland, Y. V. Brun, J. P. Reilly, J Chromatogr B Analyt Technol Biomed Life Sci 2002, 782, 363.

[12] E. D. Dodds, H. J. An, P. J. Hagerman, C. B. Lebrilla, J Proteome Res 2005, 5, 1195.

[13] E. D. Dodds, B. H. Clowers, P. J. Hagerman, C. B. Lebrilla, Anal Biochem 2008, 372, 156.

[14] W. E. Wolski, M. Farrow, A. K. Emde, H. Lehrach, M. Lalowski, K. Reinert, Proteome Sci 2006, 4, 18.

[15] C. Bruce, M. A. Shifman, P. Miller, E. E. Gulcicek, Anal Chem 2006, 78, 4374.

[16] W. D. Lehmann, A. Bohne, C. W. von der Lieth, J. Mass Spectrom 2000, 35, 1335.

[17] S. Pourshahian, P. A. Limbach, J. Mass Spectrom 2008, 43, 1081.

[18] M. Kirchner, W. Timm, P. Fong, P. Wangemann, H. Steen, Bioinformatics 2010, 26, 791.

[19] J. A. Vizcaino, E. W. Deutsch, R. Wang, A. Csordas, F. Reisinger, D. Rios, J. A. Dianes, Z. Sun, T. Farrah, N. Bandeira, P. A. Binz, I. Xenarios, M. Eisenacher, G. Mayer, L. Gatto, A. Campos, R. J. Chalkley, H. J. Kraus, J. P. Albar, S. MartinezBartolome, R. Apweiler, G. S. Omenn, L. Martens, A. R. Jones, H. Hermjakob, Nat Biotechnol 2014, 32, 223. 
Figure 1

A
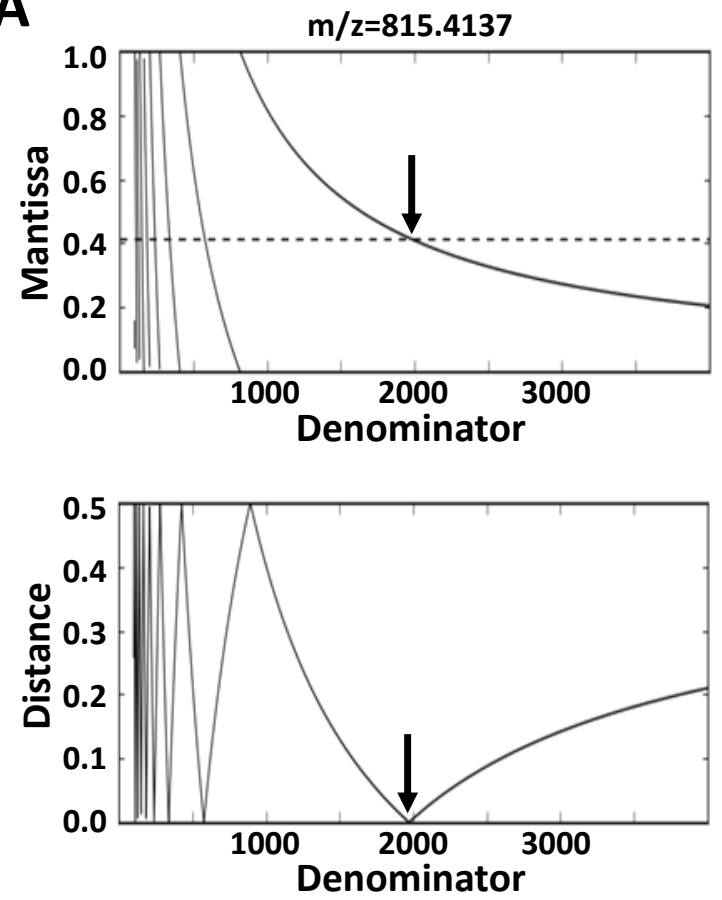

B
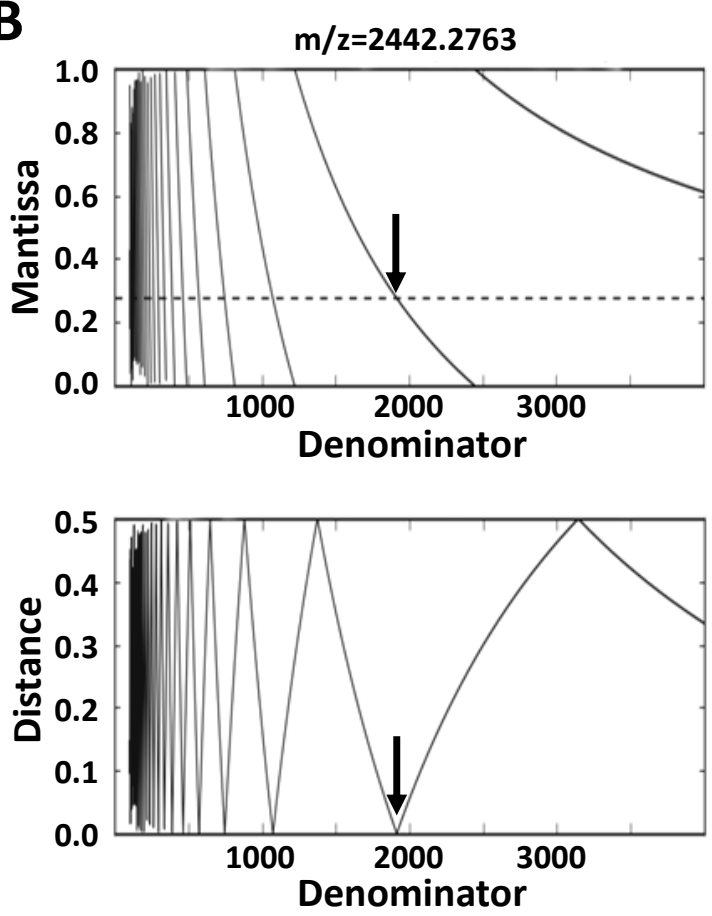
Figure 2
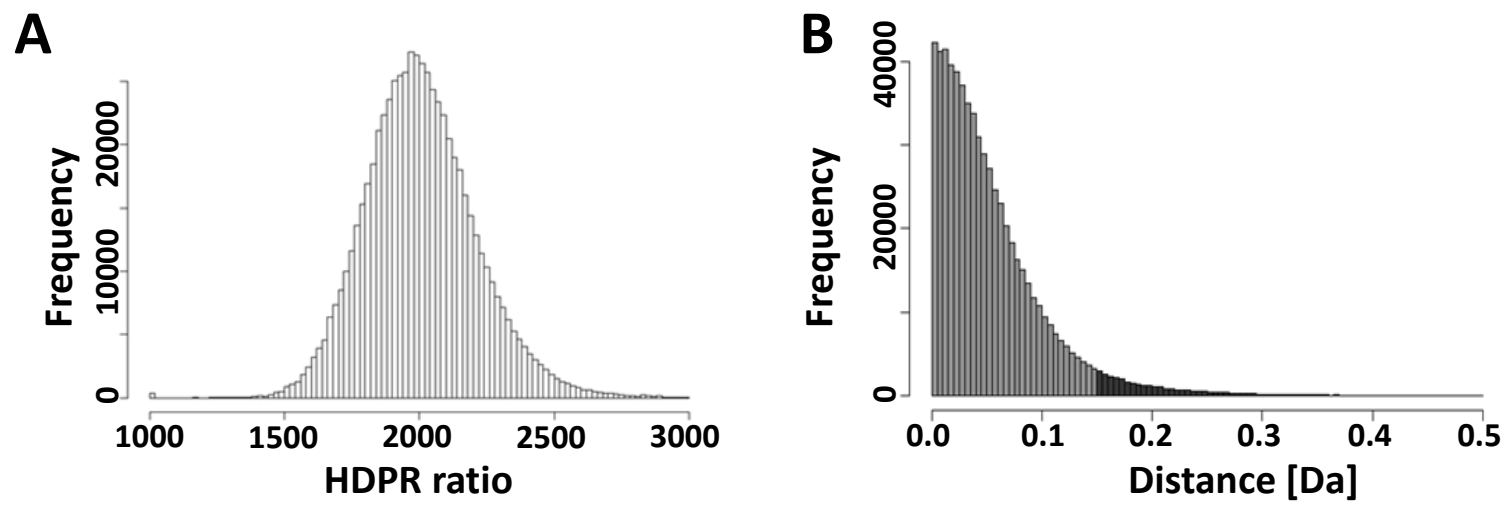
Figure 3
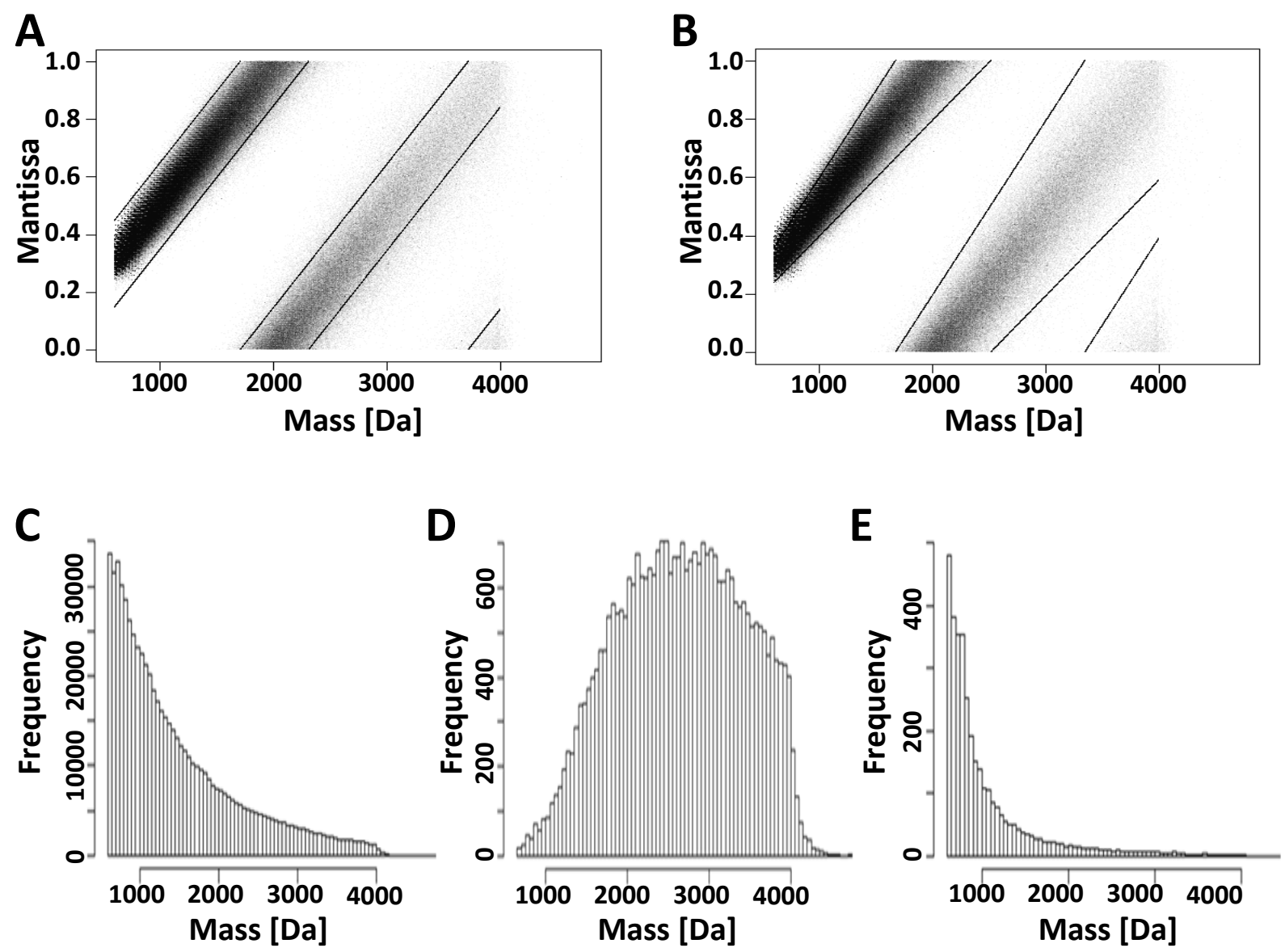
Figure 4

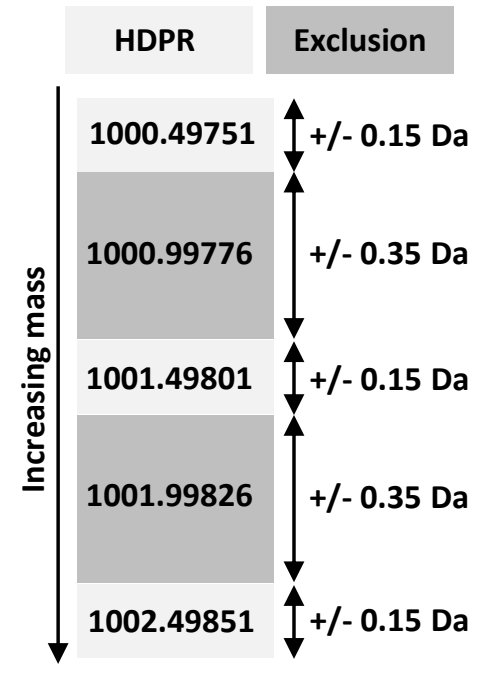

